\title{
Growth and Characterization of (110) InAs Quantum Well Heterostructures by Transmission Electron Microscopy and Electron Channeling Contrast Imaging.
}

\author{
Michael B. Katz ${ }^{1,2}$, Mark E. Twigg ${ }^{1}$, Adrian A. Podpirka ${ }^{1,2}$, Mike Hernandez ${ }^{3}$, Shawn Mack ${ }^{1}$, and Brian \\ R. Bennett ${ }^{1}$ \\ 1. Division of Electronic Science \& Technology, US Naval Research Laboratory, Washington, DC, USA \\ 2. Post-Doctoral Research Associate, National Research Council, Washington, DC, USA \\ 3. Nanotechnology Systems Division, Hitachi High Technologies America, Inc., Clarksburg, MD, USA
}

InAs quantum wells grown in the (110) direction are an important materials system in the field of spin systems. The major hurdle in realizing such devices is structural defects arising from the large mismatch, $7.2 \%$, between the InAs film and its GaAs substrate. Thus, we embark on a study utilizing transmission electron microscopy (TEM) and electron channeling contrast imaging (ECCI) to understand the density and nature of these defects.

Films were grown by molecular beam epitaxy on a GaAs (110) substrate, with a $15 \mathrm{~nm}$ InAs quantum well between two layers of GaAlSb. Electron diffraction and x-ray reciprocal space mapping reveal that the film stack is tilted about $2.1^{\circ}$ about the in-plane [0-11] axis with respect to the substrate. The defect most likely responsible for this tilt is the (a/2)[110]-type Lomer edge dislocation, which, as a misfit dislocation, provides the primary mechanism for interfacial strain relief. Enough of these parallel Lomer edge dislocations of the same polarity would account for the film tilt, as is the case in a low-angle tilt grain boundary. Cross-sectional TEM images, as shown in Figure 1, confirm the presence of such misfit dislocations at the necessary density corresponding to a separation of 5-10 $\mathrm{nm}$. In contrast, images along the orthogonal [100] zone axis, also in Figure 1, show no such population of misfit dislocations, confirming that the strain relief mechanism is highly anisotropic with respect to the growth plane.

A population of inclined microtwin-like planar defects along the (-111) plane are also present in the film. They are variously 1-3 nm thick and originate from the film-substrate interface, implying that they are seeded at the very early stages of growth. TEM images of typical examples are shown in Figure 2. Since they almost universally belong to one set of (111)-type planes in the regions examined, we surmise that these inclined microtwin arrays contribute to both the strain relief and the tilting of film relative to the substrate. $[1,2]$

We also utilized ECCI in order to get a larger view of the dislocations in the heterostructure. This technique allows the imaging of perturbations from the nominal crystal structure over a large set of length scales. Both backscatter and forescatter geometries were used in the ECCI acquisition, and are shown in Figure 3, in which microtwins were starkly visible in the ECC images. Indeed, they ran solely along the [011] direction. Through ECCI, a threading dislocation density of approximately $10^{9} \mathrm{~cm}^{-2}$ could be measured.

[1] R. Lihl et al., J. Microsc. 118 (1980), p. 89

[2] F. Ernst and P. Pirouz, J. Mater. Res. 4 (1989), p. 834. 


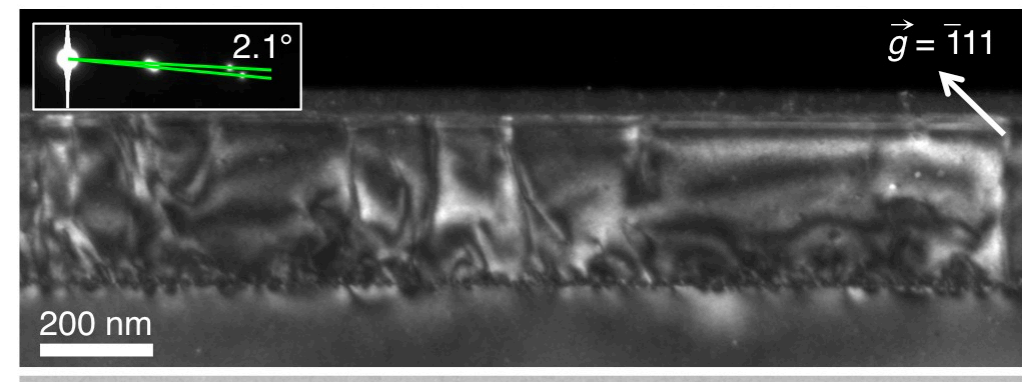

$200 \mathrm{~nm}$

Figure 1. Cross-sectional TEM images showing (top) a high density of interfacial misfit dislocations when viewed along the [100] zone axis, but (bottom) very few when viewed along the [011] zone axis. Inset is part of the diffraction pattern showing the rotation between film and substrate.

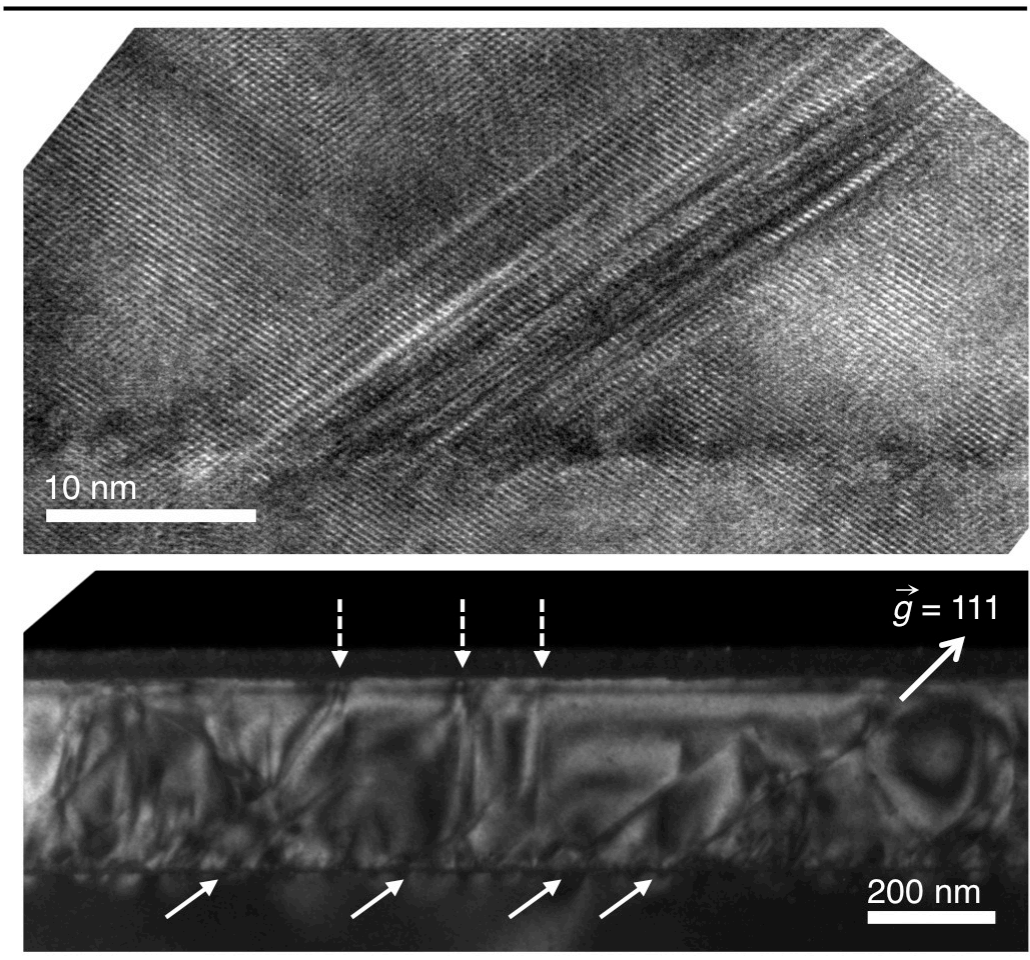

Figure 2. Several microtwin bands exist within the film. A high resolution image (top) shows a microtwin band originating at the film-substrate interface, with (bottom) a wider view showing their prevalence throughout the film.
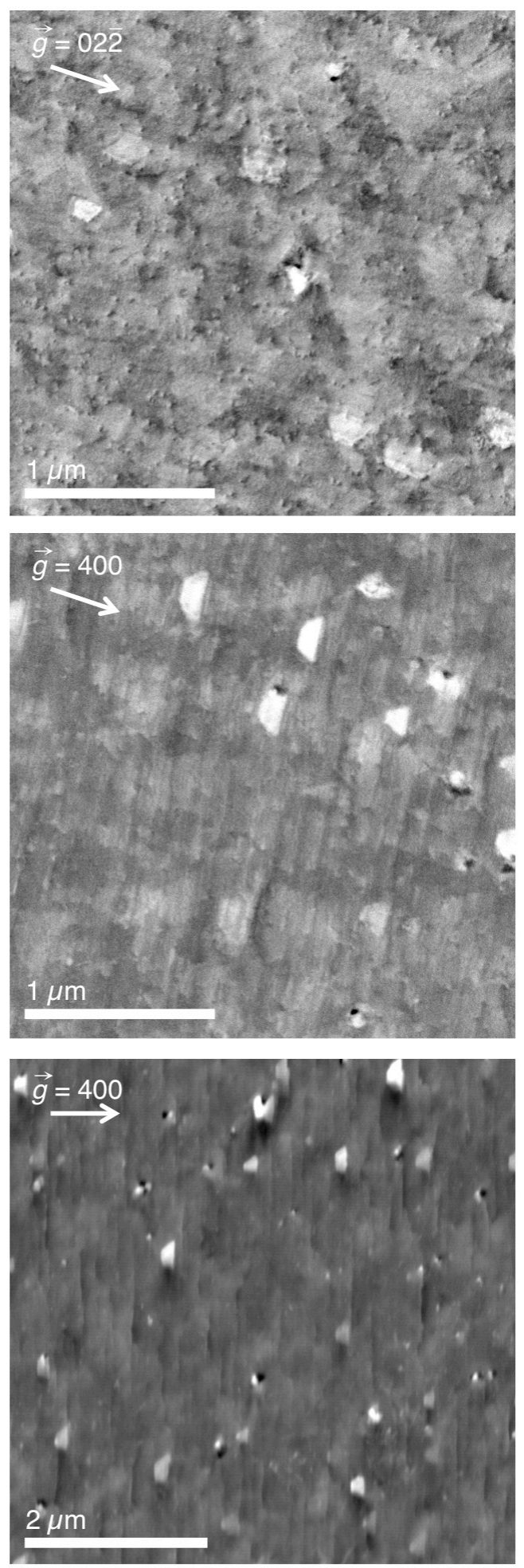

Figure 3. $\mathrm{ECCl}$ images of the film surface under various diffraction conditions show (top) threading dislocations, (middle) microtwins within the film, and (bottom) the subset of microtwins impinging the film surface. 